\title{
Polymerization of $\boldsymbol{N}$-Phenylmaleimide with Organoaluminum Initiators
}

\author{
Tokio Hagiwara, * Yasushi Sekino, Hiroshi Hamana, \\ and Tadashi NARITA \\ Department of Environmental Engineering, Saitama Institute of Technology, \\ 1690 Fusaiji, Okabe, Saitama 369-02, Japan
}

(Received September 3, 1993)

\begin{abstract}
Organoaluminum compounds-triethylaluminum (TEA), diethylaluminum chloride (DEAC), and ethylaluminum dichloride (EADC)-showed fair reactivities for polymerization of $N$-phenylmaleimide ( $N$-PMI). The polymerization probably proceeded through an anionic mechanism. The initiator systems prepared by the reactions of organoaluminums with diphenylamine (DPA) brought about appreciable effects on polymer yield depending on the molar ratio of an organoaluminum to the amine. GPC profiles showed that poly $(N$-PMI) prepared from those initiators had a high molecular weight part with fairly narrow molecular weight distribution and a low molecular weight part consisting of a dimer and trimer. The addition of 1-methylimidazole (MIm) to organoaluminum/DPA systems gave rise to increase of polymer yield and great change of GPC profile-broadening of molecular weight distribution with the disappearance of the low molecular weight part-.

KEY WORDS Organoaluminum / Diphenylamine / $N$-Substituted maleimide / $N$-Phenylmaleimide / Polymerization / Poly $(N$-Phenylmaleimide) / Anionic mechanism /
\end{abstract}

Polymerization of $N$-substituted maleimides recently has come to be intensively studied. ${ }^{1-9}$ In the last decade, a large number of $N$ substituted maleimides were synthesized and examined for polymerization reactivities, and properties of the obtained poly $(N$-substituted maleimide)s and/or their copolymers, including chiropitcal and thermal properties. $5,7,9,10$

On the anionic polymerization, polymerization reactivities of $N$-substituted maleimides for several initiators were investigated in detail. $^{2,4}$ Alkali tert-butoxides such as potassium tert-butoxides exhibited extremely high activity for the anionic polymerization of $N$-substituted maleimides, while Grignard reagents as relatively stronger anionic initiators showed lower reactivity. ${ }^{2}$ Some organozinc compounds and ate complexes were also active initiators but their polymerization reactivities were somewhat different from usual basic initiators such as alkali metal alcoholates. ${ }^{3}$
Various organoaluminum compounds play important roles in polymerization as initiators or catalysts. ${ }^{11}$ Some of them exhibit unique polymerization reactivities unable to be achieved by the other initiators. For instance, an aluminum porphyrin is effective to initiate "immortal" polymerization of epoxides. ${ }^{12}$

However, the polymerization reactivities of $\mathrm{N}$-substituted maleimides for organoaluminum initiators have not been investigated at all. In this paper, the polymerization reactivities of organoaluminum compounds are studied in detail.

\section{EXPERIMENTAL}

All experiments related to polymerization were carried out under purified nitrogen atmosphere to exclude the effects of oxygen and moisture. 


\section{Reagents}

$N$-PMI was purified as previously described. $^{2-4,6,7}$ THF and toluene (Tol.) and dichloromethane were dried and purified by the usual methods. Triethylaluminum (TEA), Diethylaluminum chloride (DEAC), and ethylaluminum dichloride (EADC) were purified by distillation under reduced pressure in nitrogen atmosphere. Boron trifluoride etherate, trifluoroacetic acid, and tin(IV) chloride were purified by distillation before use. Diphenylamine (DPA) was purified by recrystallization from hexane. 1-Methylimidazole (MIm) was dried by refluxing over calcium hydride and distilled under reduced pressure in nitrogen atmosphere.

Preparation of Organoaluminum/DPA and Organoaluminum/DPA/MIm Systems (Modified Organoaluninum Initiators)

In a flask with a three way cock toluene and an organoaluminum were placed. A definite amount of toluene solution of DPA with determined concentration was added dropwise carefully to the solution with stirring, and the reaction mixture was kept at $70^{\circ} \mathrm{C}$ for $1 \mathrm{~h}$ with continuous stirring. The reaction mixtures prepared were yellow homogeneous solutions for all molar ratios of organoaluminums to DPA. The species formed in these reaction mixture were compounds having aluminum amide structures.

An organoaluminum/DPA/MIm system was prepared by the addition of a definite amount of MIm to an above reaction mixture.

\section{Polymerization}

Polymerization was carried out in a sealed ampoule which was carefully flame dried. A solvent and $N$-PMI solution were placed into the ampoule and kept at a determined temperature. Then initiator solution was added there and the ampoule was fused and sealed. After a definite time, the reaction mixture was poured into a large amount of a mixture of ether-methanol $(3: 1 \mathrm{v} / \mathrm{v})$, and the polymer precipitated was isolated by filtration, washed with methanol three times, and then dried thoroughly above $100^{\circ} \mathrm{C}$ in vacuo. The structure of the resulting poly( $N$-PMI) was confirmed by ${ }^{1} \mathrm{H}$ NMR and IR.

\section{Measurements}

${ }^{1} \mathrm{H}$ NMR spectra were recorded on a JEOL GSX-270 FT-NMR spectrometer using deuterated dimethyl sulfoxide or chloroform as solvent. IR spectra were measured on a JASCO IR-700 spectrometer. Gel permeation chromatograms were measured with a TOSOH HLC-802A apparatus at $38^{\circ} \mathrm{C}$ with TSK gel G40000HXL-G3000HXL-G2000HXL column series using THF as eluent (flow rate

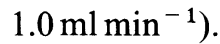

\section{RESULTS AND DISCUSSION}

\section{Polymerization with TEA, DEAC, and EADC \\ (Unmodified Organoaluminum Initiators)}

The results of polymerization of $N$-PMI with three unmodified organoaluminum compounds are summarized in Table I. Each organoaluminum is active for polymerization of $N$-PMI $(\sim 38.9 \%)$ except polymerization at low temperature, while reactivity as an initiator is very low compared to those of alkali metal tert-butoxides, ${ }^{2,4}$ typical anionic initiators for the polymerization of $\mathrm{N}$-PMI.

In polymerization with TEA as an initiator, the yield of poly( $N$-PMI) increased with polymerization time and ratio of TEA to $N$-PMI. These suggest that the polymerization of $N$-PMI with TEA is of a somewhat living nature.

Organoaluminum compounds are Lewis acids $^{13,14}$ and in some case behave as cationic initiators for several monomers such as tetrahydrofuran (THF). ${ }^{15}$ The fact that the polymerization takes place even in dichloromethane which may undergo nucleophilic substitution with some basic nucleophiles, suggests that polymerization with organoaluminum proceeds through a cationic or radical 
mechanism. However, reactivity of the vinylene group in $N$-PMI to an electrophile is considered to be much reduced by two carbonyl groups. In fact, typical cationic initiators such as boron trifluoride etherate, trifluoroacetic acid, and tin(IV) chroride were not reactive at all in dichloromethane for the addition polymerization of $N$-PMI in contrast with anionic initiators with which polymerization easily takes place in THF or toluene. ${ }^{4}$ With alkali metal tert-butoxide as an initiator, polymerization takes place at only the vinylene group in $N$-PMI and no appreciable side reaction occurs. In ${ }^{1} \mathrm{H}$ and. ${ }^{13} \mathrm{C}$ NMR spectra of poly $(N-\mathrm{PMI})$ produced with organoaluminum initiators, the signals due to vinylene group in $N$-PMI disappeared and new signals derived from methine groups in the imide ring appeared. The NMR spectra of poly $(N$-PMI) were almost the same as those with potassium tert-butoxide except for absorptions derived from initiator fragment, which can be assigned to ethyl group. This suggests that for polymerization with organoaluminum itself, initiation takes place by the addition of the ethyl fragment to the vinylene group, and polymerization proceeds only by opening of vinylene double bonds. ${ }^{4}$

These polymerizations are very slow compared to those with alkali metal tertbutoxides. $^{2,4}$ For the anionic polymerization of $N$-PMI, the polymerization rate is considerably influenced by the counter cation. ${ }^{4}$ The anionic polymerization with alkaline earth metal counter cation such as magnesium cation, polymerization proceeds much slower than that with alkali metal such as potassium cation. $^{2}$ In the polymerization of $N$-PMI with organoaluminum initiators, probably similar counter cation effect is also operative.

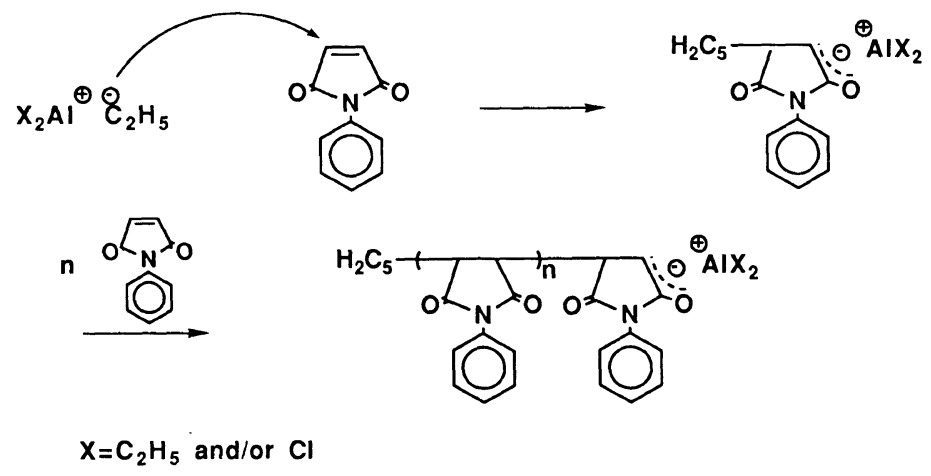

Elemental analyses and IR spectra proved that no chlorine was incorporated in the polymer obtained in dichloromethane used as a solvent. This implies that abstraction of chlorine atoms, which can occur if any radical intermediates are generated in the polymerization system, does not occur.

It is thus considered that the polymerization of $N$-PMI with organoaluminum compounds is, at least for the polymerization with TEA, dominated by the anionic or anionic coordination mechanism through aluminum enolate species, which is not so strong as to be basic and nuculeophilic to attack solvent molecule having chlorine atom to terminate polymerization or give rise to chain transfer.

Figure 1 shows GPC traces of poly( $N$-PMI) produced with three different unmodified organoaluminum initiators. All of these consist of high molecular weight and oligomer (dimer and trimer) parts. Number average molecular weights calibrated by polystyrene standard are rather low as shown in Table I, and molecular weight distributions were substantially broad due to oligomer parts: $\bar{M}_{n}=1.1 \times 10^{3},\left(\bar{M}_{w}\right)$ $\bar{M}_{n}=7.1$ ) for TEA (run 2), $\bar{M}_{n}=1.6 \times 10^{3}$, 
Table I. Polymerization of $N$-PMI with organoaluminum compounds as initiators

\begin{tabular}{|c|c|c|c|c|c|c|c|}
\hline Run & Org. Al & {$[N$-PMI $] /[$ Org. Al] } & Solv. & $\frac{\text { Temp. }}{{ }^{\circ} \mathrm{C}}$ & $\begin{array}{l}\text { Time } \\
\text { day }\end{array}$ & $\frac{\text { Yield }}{\%}$ & $\begin{array}{c}\bar{M}_{n}^{\mathrm{d}} \\
\times 10^{-3}\end{array}$ \\
\hline $1^{\mathrm{a}}$ & TEA & 25 & Tol. & 40 & 7 & 6.9 & $\mathrm{NM}^{\mathrm{e}}$ \\
\hline $2^{a}$ & TEA & 25 & Tol & 40 & 14 & 15.7 & 1.1 \\
\hline $3^{\mathrm{b}}$ & TEA & 67 & $\mathrm{CH}_{2} \mathrm{Cl}_{2}$ & 22 & 4 & 1.0 & NM \\
\hline $4^{a}$ & TEA & 60 & $\mathrm{CH}_{2} \mathrm{Cl}_{2}$ & 22 & 4 & 3.5 & NM \\
\hline $5^{\mathrm{c}}$ & TEA & 31 & $\mathrm{CH}_{2} \mathrm{Cl}_{2}$ & 22 & 4 & 9.0 & 0.8 \\
\hline $6^{b}$ & TEA & 20 & $\mathrm{CH}_{2} \mathrm{Cl}_{2}$ & 22 & 4 & 19.2 & 1.2 \\
\hline $7^{\mathrm{b}}$ & TEA & 14 & $\mathrm{CH}_{2} \mathrm{Cl}_{2}$ & 22 & 4 & 38.9 & 1.2 \\
\hline $8^{b}$ & TEA & 14 & $\mathrm{CH}_{2} \mathrm{Cl}_{2}$ & 0 & 4 & 0 & - \\
\hline $9^{a}$ & DEAC & 25 & Tol. & 40 & 7 & 10.2 & 1.6 \\
\hline $10^{\mathrm{a}}$ & EADC & 25 & Tol. & 40 & 7 & 7.4 & 1.0 \\
\hline \multicolumn{8}{|c|}{$\begin{array}{l}\text { a }[N-\mathrm{PMI}]=2.9 \times 10^{-1}\left(\mathrm{moll}^{-1}\right) \\
\text { b }[N-\mathrm{PMI}]=2.2 \times 10^{-1}\left(\mathrm{moll}^{-1}\right) \\
\text { c }[N-\mathrm{PMI}]=2.0 \times 10^{-1}\left(\mathrm{moll}^{-1}\right) \\
\text { d } \text { From GPC eluogram calibrated by the polystyrene standard. } \\
\text { e Not measured. }\end{array}$} \\
\hline
\end{tabular}

with TEA

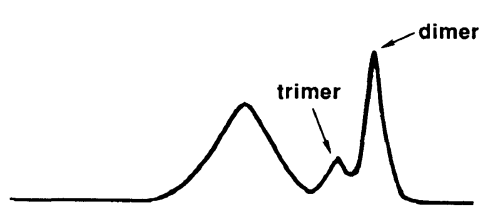

with DEAC

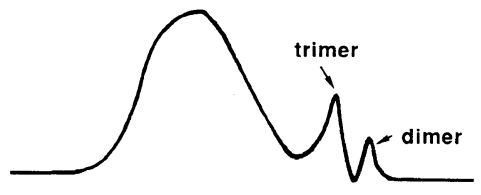

with EADC

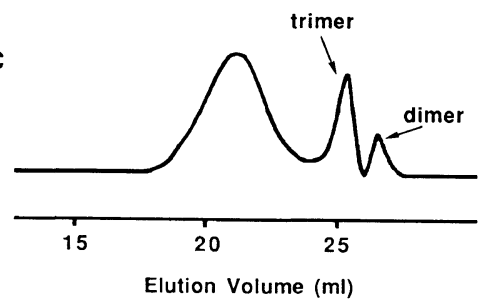

Figure 1. GPC profiles of poly $(N-\mathrm{PMI}) \mathrm{s}$ obtained by initiation with three organoaluminum compounds (TEA, triethylaluminum; DEAC, diethylaluminum chloride; EADC, ethylaluminum dichloride). Polymerization condition: in toluene, at $40^{\circ} \mathrm{C}$, for 7 days.
$\left(\bar{M}_{w} / \bar{M}_{n}=5.8\right)$ for DEAC (run 9), and $\bar{M}_{n}=1.0 \times 10^{3}\left(\bar{M}_{w} / \bar{M}_{n}=6.1\right)$ for EADC (run 10). However, the molecular weights of high molecular weight parts are $M_{n}=1.9 \times 10^{3}$, $4.7 \times 10^{3}$, and $2.6 \times 10^{3}$ for high molecular weight peaks of the polymers with TEA, DEAC, and EADC, respectively. Molecular weight distributions of high molecular weight parts are unimodal and fairly narrow for polymerizations with TEA and EADC $\left(\bar{M}_{w}\right)$ $\bar{M}_{n}=1.3$ for each), while in GPC traces of high molecular weight parts of poly( $N$-PMI) with DEAC, a shoulder is observed and thus the molecular weight distribution is relatively broad $\left(\bar{M}_{w} / \bar{M}_{n}=2.8\right)$. Similar GPC patterns of poly $(N$-PMI) initiated with TEA and EADC were observed in those of poly $(N-\mathrm{PMI})$ prepared with organozinc initiators ${ }^{3}$.

\section{Polymerization with Organoaluminum initiators Modified by Amines}

The initiator system prepared by modification of TEA with DPA is of interest. It can polymerize MMA while unmodified TEA is unable to initiate polymerization. ${ }^{16}$ Initiator systems prepared by modification of organoaluminums with amines or water were also used 
Table II. Polymerization of $N$-PMI with TEA/DPA and TEA/DPA/MIm systems ${ }^{\mathrm{a}}$

\begin{tabular}{|c|c|c|c|c|c|c|}
\hline \multirow{2}{*}{ Run } & TEA/DPA & MIm added & TEA/MIm & Time & Yield & \multirow{2}{*}{$\begin{array}{c}\bar{M}_{n}^{\mathbf{b}} \\
\times 10^{-3}\end{array}$} \\
\hline & $\mathrm{mol} \mathrm{mol}^{-1}$ & $\mathrm{mmol}$ & $\mathrm{mol} \mathrm{mol}^{-1}$ & day & $\%$ & \\
\hline 1 & $1 / 0.5$ & - & - & 7 & 12.4 & 1.0 \\
\hline 2 & $1 / 0.5$ & - & - & 14 & 22.7 & 1.6 \\
\hline 3 & $1 / 1$ & - & - & 7 & 16.4 & 1.4 \\
\hline 4 & $1 / 1$ & - & - & 14 & 22.7 & 2.0 \\
\hline 5 & $1 / 1$ & 0.12 & $1 / 1$ & 7 & 6.9 & 1.6 \\
\hline 6 & $1 / 1$ & 1.2 & $1 / 10$ & 7 & 22.4 & 2.3 \\
\hline 7 & $1 / 1.5$ & - & - & 7 & 3.4 & $\mathrm{NM}^{\mathrm{c}}$ \\
\hline 8 & $1 / 1.5$ & - & - & 14 & 13.9 & 0.8 \\
\hline 9 & $1 / 2$ & - & - & 7 & 3.1 & NM \\
\hline 10 & $1 / 2$ & - & - & 14 & 6.7 & NM \\
\hline 11 & $1 / 2$ & 0.12 & $1 / 1$ & 7 & 5.7 & NM \\
\hline 12 & $1 / 2$ & 1.2 & $1 / 10$ & 7 & 22.0 & 2.2 \\
\hline 13 & $1 / 3$ & - & - & 7 & 2.9 & NM \\
\hline 14 & $1 / 3$ & - & - & 14 & 7.1 & NM \\
\hline 15 & $1 / 3$ & 0.12 & $1 / 1$ & 7 & 10.5 & 1.8 \\
\hline 16 & $1 / 3$ & 1.2 & $1 / 10$ & 7 & 20.1 & 2.1 \\
\hline
\end{tabular}

${ }^{\mathrm{a}}[N-\mathrm{PMI}]=2.9 \times 10^{-1}\left(\mathrm{moll}^{-1}\right),[\mathrm{Al}]=1.2 \times 10^{-2}\left(\mathrm{moll}^{-1}\right)$, in toluene, at $40^{\circ} \mathrm{C}$.

b From GPC eluogram calibrated by the polystyrene standard.

${ }^{c}$ Not measured.

Table III. Polymerization of $N$-PMI with DEAC/DPA and DEAC/DPA/MIm systems ${ }^{a}$

\begin{tabular}{|c|c|c|c|c|c|}
\hline \multirow{2}{*}{ Run } & DEAC/DPA & MIm added & DEAC/MIm & Yield & \multirow{2}{*}{$\begin{array}{c}\bar{M}_{n}^{\mathrm{b}} \\
\times 10^{-3}\end{array}$} \\
\hline & $\mathrm{mol} \mathrm{mol}^{-1}$ & $\mathrm{mmol}$ & $\mathrm{mol} \mathrm{mol}^{-1}$ & $\%$ & \\
\hline 1 & $1 / 1$ & - & - & 0.4 & $\mathrm{NM}^{\mathrm{c}}$ \\
\hline 2 & $1 / 1$ & 0.12 & $1 / 1$ & 0.7 & NM \\
\hline 3 & $1 / 1$ & 1.2 & $1 / 10$ & 21.4 & 2.2 \\
\hline 4 & $1 / 2$ & - & - & 1.6 & NM \\
\hline 5 & $1 / 2$ & 0.12 & $1 / 1$ & 0.2 & NM \\
\hline 6 & $1 / 2$ & 1.2 & $1 / 10$ & 16.9 & 1.7 \\
\hline
\end{tabular}

${ }^{\mathrm{a}}[N$-PMI $]=2.9 \times 10^{-1}\left(\mathrm{moll}^{-1}\right),[\mathrm{Al}]=1.2 \times 10^{-2}\left(\mathrm{moll}^{-1}\right)$, in toluene, at $40^{\circ} \mathrm{C}, 7$ days.

${ }^{b}$ From GPC eluogram calibrated by the polystyrene standard.

c Not measured.

as polymerization catalysts for several cyclic monomers such as THF and propylene oxide. $^{12,15,17}$

The results of polymerization of $N$-PMI with modified organoaluminums prepared by reactions with DPA or DPA/MIm are shown in Tables II for TEA, III for DEAC, and IV for EADC. The initiators prepared from the reaction of TEA with DPA being less than or equal to unity in molar ratios for DPA/TEA give higher polymer yields $(22.7 \%$ in toluene at $40^{\circ} \mathrm{C}$ for 14 days) than TEA itself $(15.7 \%$ under the same polymerization condition-see Table II-). Lower polymer yields (6.7$13.9 \%$ ) are obtained when the DPA/TEA molar ratio is more than unity. The modified organoaluminum prepared by the equimolar reaction of DPA with TEA is optimum initiator in terms of polymer yield in the TEA/DPA system. 
Table IV. Polymerization of N-PMI with EADC/DPA and EADC/DPA/MIm systems ${ }^{\mathbf{a}}$

\begin{tabular}{|c|c|c|c|c|c|}
\hline \multirow{2}{*}{ Run } & EADC/DPA & MIm added & EADC/MIm & Yield & \multirow{2}{*}{$\begin{array}{c}\bar{M}_{n}^{\mathrm{b}} \\
\times 10^{-3}\end{array}$} \\
\hline & $\mathrm{mol} \mathrm{mol}^{-1}$ & $\mathrm{mmol}$ & $\mathrm{mol} \mathrm{mol}^{-1}$ & $\%$ & \\
\hline 1 & $1 / 1$ & - & - & 0.1 & $\mathrm{NM}^{\mathrm{c}}$ \\
\hline 2 & $1 / 1$ & 0.12 & $1 / 1$ & 0.3 & NM \\
\hline 3 & $1 / 1$ & 1.2 & $1 / 10$ & 25.1 & 2.3 \\
\hline
\end{tabular}

${ }^{a}[N-\mathrm{PMI}]=2.9 \times 10^{-1}\left(\mathrm{moll}^{-1}\right),[\mathrm{Al}]=1.2 \times 10^{-2}\left(\mathrm{moll}^{-1}\right)$, in toluene, at $40^{\circ} \mathrm{C}, 7$ days.

b From GPC eluogram calibrated by the polystyrene standard.

c Not measured.

For DEAC (Table III, run 1 and 4) and EADC (Table IV, run 1), simple modifications to organoaluminum compounds by DPA do not improve but rather lower initiator activity for the polymerization of $N$-PMI with respect to the polymer yield.

Organoaluminum species having two $\mathrm{Al}-\mathrm{C}$ and one $\mathrm{Al}-\mathrm{N}$ bond for one aluminum atom are the most reactive as $N$-PMI polymerization initiators. This is probably due to the activation of $\mathrm{Al}-\mathrm{C}$ bonds by the formation of $\mathrm{Al}-\mathrm{N}$ bonds. The structure of this species is that of a dimer of diethylaluminum diphenylamide with amide bridge. ${ }^{13}$<smiles>CC[Si]1(CC)N(c2ccccc2)[Al](C)(C)N1c1ccccc1</smiles>

GPC profile of $\operatorname{poly}(N-\mathrm{PMI})$ with the initiator system modified by only DPA is shown in Figure 2 (upper). The observed GPC pattern is not so different from that in the case of polymerization with unmodified TEA (see Figure 1), though the ratio of high molecular weight part of polymer to low one becomes a little larger by modification with DPA. The number average molecular weight (calibrated by polystyrene standard) and molecular weight distribution for whole part including oligomer are: $\bar{M}_{n}=1.4 \times 10^{3}$ and $\bar{M}_{w} / \bar{M}_{n}=4.2$. The high molecular weight part has a somewhat broad molecular weight distribution $\left(\bar{M}_{w} / \bar{M}_{n}=1.7\right)$ compared to that with unmodified TEA,
TEA/DPA=1/1

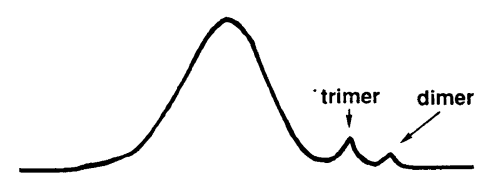

TEA/DPA/MIm=1/1/10

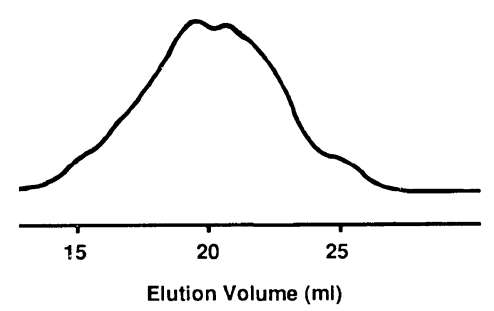

Figure 2. GPC profiles of poly(N-PMI) obtained with modified TEA initiator systems. Polymerization condition: in toluene, at $40^{\circ} \mathrm{C}$, for 7 days.

though it is also unimodal. Although both $\mathrm{Al}-\mathrm{C}$ and $\mathrm{Al}-\mathrm{N}$ could initiate the polymerization of $N$-PMI, the results obtained from Tables II-V and GPC analyses suggest that initiation by attacking of ethyl group in organoaluminum to $\mathrm{N}$-PMI molecules is dominant compared to that of aluminum amide species in these cases.

Some Lewis bases work as complexing agents and increase the reactivities of organoaluminum initiators for the anionic polymerization due to complexation with aluminum atom. ${ }^{18}$ For the polymerization of $N$-PMI initiated with organoaluminum compounds, the addition of MIm as a Lewis base of the 
third component to initiator system has considerable effect on polymer yield except for the case of TEA/DPA/MIm $=1 / 1 / 1$. The highest yield of $22 \%$ is obtained with TEA $/ \mathrm{DPA} / \mathrm{MIm}=1 / 1 / 10$ or $1 / 2 / 10$ initiator system (Table II, run 6 and 12). Polymer yields increased with the amount of MIm added for all polymerization systems.

GPC profile of poly( $N$-PMI) with TEA/ DPA/MIm systems is depicted in Figure 2 (down). The number average molecular weight (calibrated by polystyrene standard) and molecular weight distribution are $2.3 \times 10^{3}$ and $\bar{M}_{w} / \bar{M}_{n}=4.6$. The addition of MIm results in appreciable change in the GPC profile of poly $(N$-PMI), broadening and polymodality of molecular weight distribution and decrease in low molecular weight part.

These results imply that reactivity of aluminum amide for the initiation become higher probably because not only $\mathrm{Al}-\mathrm{C}$ bonds activated by $\mathrm{Al}-\mathrm{N}$ bond formation as abovementioned but $\mathrm{Al}-\mathrm{N}$ bonds are also activated in those systems by complexation of MIm with aluminum atom.

\section{CONCLUSION}

With organoaluminum compounds-TEA, DEAC, and EADC- $N$-PMI was polymerized probably through anionic process with aluminum enolate species though the reactivities of them all as initiators for anionic polymerization of $N$-PMI were not good.

Molecular weight distributions of $\operatorname{poly}(N$ PMI) prepared with unmodified organoaluminum initiators involve a high molecular weight part which have fairly narrow distribution $\left(\bar{M}_{w} / \bar{M}_{n}=1.3\right.$ for TEA and EADC) and a low molecular weight part which consists of dimer and trimer.

Modification of organoaluminum initiators by DPA brought about some effect on polymer yield. The molar ratio of an organoaluminum to the amine influenced much increase and decrease of polymer yield but did not make remarkable changes on GPC profiles of $\operatorname{poly}(N-\mathrm{PMI})$.

Considerable effects on both polymer yield and molecular weight distribution of $\operatorname{poly}(N$ PMI) were observed by the addition of MIm to the polymerization system. Appreciable increase of polymer yield and broadening of molecular weight distribution were observed in polymerization systems involving MIm.

\section{REFERENCES}

1. R. C. P. Cubbon, Polymer., 6, 419 (1965).

2. T. Hagiwara, J. Mizota, H. Hamana, and T. Narita, Makromol. Chem., Rapid Commun., 6, 169 (1985); T. Hagiwara, T. Someno, H. Hamana, and T. Narita, J. Polym. Sci., Polym. Chem. Ed., 26, 1011(1988).

3. T. Hagiwara, J. Sato, H. Hamana, and T. Narita, Makromol. Chem., 188, 1825(1985); T. Hagiwara, M. Takeda, H. Hamana, and T. Narita, Makromol. Chem., Rapid Commun., 8, 167 (1987).

4. T. Hagiwara, T. Shimizu, T. Someno, T. Yamagishi, H. Hamana, and T. Narita, Macromolecules, 21, 3324 (1988); T. Hagiwara, T. Shimizu, T. Uda, H. Hamana, and T. Narita, J. Polym. Sci., Polym. Chem. Ed., 28, 185 (1990); T. Hagiwara, T. Shimizu, H. Hamana, and T. Narita, J. Polym. Sci., Polym. Chem. Ed., 28, 2437 (1990)

5. T. Hagiwara, I. Suzuki, K. Takeuchi, H. Hamana, and T. Narita, Macromolecules, 24, 6856(1991).

6. M. Yamada, I. Takase, and T. Mishima, Kobunshi Kagaku, 23, 348 (1966); ibid., 24, 326 (1967); ibid., 26, 393 (1969); M. Yamada, I. Takase, and M. Kabayashi, ibid., 29, 144 (1972).

7. T. Hagiwara, M. Takeda, H. Hamana, and T. Narita, Macromolecules, 22, 2025 (1989); T. Hagiwara, S. Nakazawa, H. Hamana, and T. Narita, Polym. Bull., 24, 299(1990); T. Hagiwara, N. Yanaba, T. Takada, H. Hamana, and T. Narita, Polym. Bull., 26, 503 (1991).

8. H. Yamaguchi and Y. Minoura, J. Polym. Sci., A-1, 8, 929(1970); ibid., $A-1,8,1467$ (1970); N. Sakata, K. Kishiue, S. Shimada, and Y. Minoura, J. Polym, Sci., Polym. Chem. Ed., 12, 1787 (1974); T. Oishi and N. Kimura, Kobunshi Ronbunshu, 32, 380 (1975); ibid., 33, 141 (1976); ibid., 33, 685 (1976); T. Oishi, T. Ishikawa, and T. Kimura, ibid., 36, 751 (1979).

9. T. Oishi and M. Fujimoto, J. Polym. Sci., Polym. Chem. Ed., 22, 2789 (1984); T. Oishi, H. Yamasaki, and M. Fujimoto, Polym. J., 23, 795 (1991); T. Oishi, M. Iwahara, and M. Fujimoto, ibid., 23, 1409 (1991); T. Oishi, A. Kamori, and M. Fujimoto, J. Macromol. Sci., Pure Appl. Chem., A29, 231(1992); T. Oishi, and M. Fujimoto, J. Polym. Sci., Polym. Chem. Ed., 30, 
1921 (1992); T. Oishi, K. Matsusaki, and M. Fujimoto, Polym. J., 24, 1281 (1992); T. Oishi, K. Kagawa, and M. Fujimoto, Macromolecules, 26, 24 (1993).

10. Y. Okamoto, T. Nakano, H. Kobayashi, and K. Hatada, Polym. Bull., 25, 5 (1991).

11. L. Reich and A. Schindler, "Polymerization by Organometallic Compounds" Wiley Interscience, New York, N.Y., 1966, p 115 and 672.

12. T. Aida, Y. Maekawa, S. Asano, and S. Inoue, Macromolecules, 20, 1473 (1987).

13. J. J. Eisch, "Aluminum" in "Comprehensive Organometallic Chemistry,"Vol. 1, Ed. G. Wilkin- son, Pergamon, Oxford, 1982, p 555.

14. H. Imai, T. Saegusa, and J. Furukawa, Makromol. Chem., 81, 92 (1965); T. Saegusa, H. Imai, and S. Matsumoto, J. Polym. Sci., A-1, 6, 459 (1968).

15. T. Saegusa, H. Imai, and J. Furukawa, Makromol. Chem., 53, 203 (1962).

16. S. Muranishi, T. Niki, T. Kubokata, H. Yuki, and K. Hatada, Kobunshi Kagaku, 24, 198 (1967); ibid., 24, 309 (1967).

17. E. J. Vandenberg, J. Polym. Sci., 47, 486 (1960).

18. M. Ikeda, T. Hirano, and T. Tsuruta, Makromol. Chem., 150, 127 (1967). 